\title{
Caracterização colorimétrica de pisos de madeiras tropicais amazônicas
}

\author{
Ana Carolina Silva Costa $\odot^{1}$ Bárbara Luísa Corradi Pereira $\odot^{1}$ Mirian Fátima de Almeida Silva $\odot^{1}$ \\ Elaine Cristina Lengowski ${ }^{1}$ Aylson Costa Oliveira ${ }^{1 *}$
}

${ }^{1}$ Universidade Federal do Mato Grosso, Av. Fernando Corrêa da Costa, 2367, Boa Esperança, CEP 78060-900, Cuiabá, MT, Brasil

\begin{abstract}
Original Article
*Corresponding author: aylsoncosta@gmail.com

Keywords:

Color

Basic Density

Correlation

Palavras-chave:

Cor

Densidade básica

Correlação

Received in

$2021 / 06 / 27$

RESUMO: A cor da madeira é uma característica decisiva na escolha de pisos pelos consumidores. O objetivo deste estudo foi fazer a caracterização colorimétrica de pisos de cinco espécies tropicais amazônicas: Dipteryx odorata (cumaru), Handroanthus spp. (ipê), Hymenaea courbaril (jatobá), Astronium lecointei (muiracatiara) e Bowdichia virgilioides (sucupira-preta), bem como correlacionar os parâmetros colorimétricos com a densidade básica da madeira. A caracterização colorimétrica foi determinada na face superior tangencial dos pisos, obtendo-se os parâmetros colorimétricos L* (luminosidade), a* (coordenada cromática verde-vermelho) e $\mathrm{b}^{*}$ (coordenada cromática azul-amarelo), com base no sistema CIEL*a*b*, e os parâmetros C (saturação) e $h^{*}$ (ângulo de tinta) foram calculados. Os resultados foram submetidos à análises de variância e ao teste de Tukey $(p \leq 0,05)$ para verificar diferenças entre as espécies e avaliou-se a correlação de Pearson entre os parâmetros colorimétricos e a densidade básica da madeira. Os parâmetros colorimétricos variaram entre as espécies, com $\mathrm{L}^{*}$ de 47,43 a 63,$08 ; a^{*}$ de 6,50 a 13,$52 ; b^{*}$ de 17,74 a 26,16 ; C de 18,89 a 29,31; e, h* de 61,56 a 69,86 e esses apresentaram baixa variação dentro da mesma espécie. Conclui-se que os pisos de madeira das espécies cumaru, ipê e sucupira-preta são escuros e os de jatobá e muiracatiara, claros. Há uma correlação inversa entre a densidade básica e a luminosidade $\left(\mathrm{L}^{*}\right)$ das madeiras avaliadas. A colorimetria pode ser utilizada como critério auxiliar de classificação de madeiras destinadas à produção de pisos.
\end{abstract}

Accepted on

2021/09/27

Published in 2021/10/11

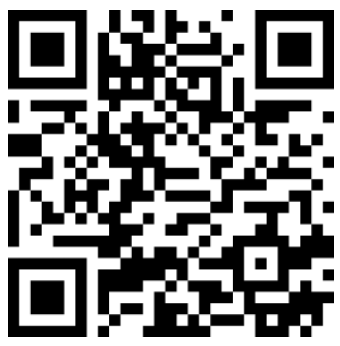

DOI:

https://doi.org/10.34062/afs. v8i3. 12533

\section{(cc) BY}

\section{Colorimetric characterization of Amazonian tropical hardwood floors}

\begin{abstract}
Wood color is a decisive characteristic in the choice of flooring by consumers. The objective of this study was to perform the colorimetric characterization of floors from five tropical Amazonian species: Dipteryx odorata (“cumaru”), Handroanthus sp. (“ipê"), Hymenaea courbaril ("jatobá"), Astronium lecointei ("muiracatiara"), and Bowdichia virgilioides ("sucupirapreta"), as well as to correlate the colorimetric parameters with the basic density of the wood. The colorimetric characterization was determined on the upper tangential face of the floors, obtaining the colorimetric parameters $\mathrm{L}^{*}$ (brightness), $a^{*}$ (green-red chromatic coordinate) and $b^{*}$ (blue-yellow chromatic coordinate), based on the CIEL*a*b* system, and the parameters $\mathrm{C}$ (saturation) and $\mathrm{h} *$ (paint angle) were calculated. The results were submitted to analysis of variance and Tukey's test $(p \leq 0.05)$ to verify differences between species, and Pearson's correlation between colorimetric parameters and wood basic density was evaluated. The colorimetric parameters varied among the species, with $\mathrm{L}^{*}$ from 47.43 to 63.08 ; $a^{*}$ from 6.50 to 13.52 ; $b^{*}$ from 17.74 to 26.16 ; $\mathrm{C}$ from 18.89 to 29.31; and, $\mathrm{h} *$ from 61.56 to 69.86 and these showed low variation within the same species. We conclude that the wood floors of the species "cumaru", "ipê", and "sucupira-preta" are dark and those of "jatobá" and "muiracatiara" are light. There is an inverse correlation between the basic density and the luminosity ( $\mathrm{L}^{*}$ ) of the evaluated woods. Colorimetry can be used as an auxiliary criterion for classifying wood for flooring production.
\end{abstract}




\section{Introdução}

Os pisos de madeira possuem destaque econômico no segmento de Produtos de Maior Valor Agregado (PMVA) do setor industrial madeireiro brasileiro. A produção estimada, no ano de 2018, foi de 8,5 milhões de metros quadrados, destinados principalmente para o mercado externo, o que resultou no valor exportado de US\$ 406 milhões em pisos de madeira dos tipos maciço (peças sólidas padronizadas) e engenheirados (camadas internas de compensado e parte superior de madeira maciça nobre) (Associação Brasileira da Indústria de Madeira Processada Mecanicamente 2019).

Os principais estados brasileiros exportadores de pisos de madeira são o Pará, Paraná, Mato Grosso, Santa Catarina e São Paulo (Associação Brasileira da Indústria de Madeira Processada Mecanicamente 2019). Destaca-se que os estados exportadores do sul e sudeste do Brasil adquirem matéria-prima da região norte para 0 beneficiamento. Assim, as espécies nativas tropicais amazônicas são as mais utilizadas para fabricação de pisos e segundo Silva et al. (2017), as espécies tropicais se destacam por seus atributos estéticos, resultantes de suas propriedades organolépticas como cor, brilho, desenho, etc, além de elevada qualidade, sendo necessário realizar estudos que caracterizem madeiras com mercado estabelecido, e buscar, por semelhança de caracteres, novas espécies.

Os pisos são amplamente utilizados em ambientes residenciais e comerciais e a sua escolha, além das propriedades tecnológicas da madeira e custos, considera a cor, propriedade sensorial, que é a primeira característica observada pelo consumidor. Por essa razão, a avaliação colorimétrica deve ser incorporada à caracterização tecnológica da madeira, para classificação e indicação das espécies madeireiras mais indicadas para determinada utilização (Barros et al. 2014), como para confecção de pisos. Além de ser utilizada para avaliar a qualidade da madeira, a cor pode ser um parâmetro para valorização dos produtos, quando, por exemplo, a cor da madeira de uma espécie não comumente comercializada se assemelha a outra já reconhecida pelo mercado (Martins et al. 2015).

As variações da cor ocorrem entre madeiras de diferentes espécies, como também dentro de uma mesma espécie e/ou indivíduo arbóreo, uma vez que essa propriedade é influenciada pela espécie, densidade básica, umidade, constituintes químicos, principalmente a quantidade e tipos de extrativos, estrutura anatômica e idade da árvore (Gierlinger et al. 2004, Silva et al. 2017). Logo, a uniformidade da cor em um lote de madeira beneficiada, pode ser um indicativo da homogeneidade dos produtos, tornando-se essencial determinar os parâmetros colorimétricos da madeira, bem como analisar sua variabilidade (Martins et al. 2015). De acordo com Moya et al. (2012), quando há uniformidade da cor da madeira, os clientes podem estar dispostos a pagar preços mais altos.

A colorimetria tem sido aplicada como ferramenta na caracterização da madeira e derivados como na avaliação da ação de intemperismo, ataque de agentes biodeterioradores, efeitos de processos de modificação térmica ou produtos de acabamento (Bonfatti Júnior et al. 2018). Além disso, mostra-se como uma técnica potencial para classificação da madeira, em linhas de produção de produtos de madeira sólida, em razão de sua rápida determinação e a possibilidade de utilização de aparelhos portáteis como os colorímetros.

Assim, a determinação dos parâmetros colorimétricos de pisos comerciais pode auxiliar na seleção de espécies potenciais para confecção de pisos. Na literatura, há diversos estudos que relatam a correlação entre os parâmetros colorimétricos da madeira e a sua densidade básica (Amorim et al. 2013, Silva et al. 2019, Sousa et al. 2019). E segundo Blanco et al. (2015), Oliveira et al. (2019) e Costa et al. (2021), na seleção de matéria-prima para a produção de pisos, a densidade da madeira é um dos principais fatores a serem considerados, pois madeiras mais densas resultam em pisos com maiores valores de resistência nos ensaios de simulação de pisos em uso, como impacto da esfera de aço cadente, atrito estático e dinâmico, carga rolante, ou seja, em ambientes de tráfego intenso sofrerão menos efeito quando da ocorrência de arraste ou queda de objetos, ou seja, terão maior durabilidade ao longo do tempo.

Assim, o objetivo deste trabalho foi realizar a caracterização colorimétrica por meio do sistema CIEL*a*b* de pisos maciços da madeira de cinco espécies tropicais amazônicas e determinar a correlação desses parâmetros com a densidade básica da madeira.

\section{Material e Métodos}

As peças de pisos de madeira maciça tipo assoalho, utilizadas para a realização deste estudo, foram fornecidas pela indústria madeireira Brasil Tropical Pisos, localizada no município de Alta Floresta, Mato Grosso. Foram utilizados pisos fabricados com madeiras de cinco espécies tropicais amazônicas, que tiveram a sua densidade básica determinada conforme Tabela 1. 
Costa et al.

Tabela 1. Espécies e densidade básica das madeiras tropicais amazônicas avaliadas

\begin{tabular}{ccc}
\hline Espécie & Nome Comum & Densidade básica*1 $\left({\left.\mathrm{g} . \mathrm{cm}^{-3}\right)}^{-3}\right.$ \\
\hline Dypteryx odorata (Aubl.) Willd & Cumaru & 0,908 \\
Handroanthus spp. & Ipê & 0,958 \\
Hymeneae courbaril Linneaeus & Jatobá & 0,847 \\
Astronium lecointei Ducke & Muiracatiara & 0,735 \\
Bowdichia virgilioides Kunth & Sucupira-preta & 0,721 \\
\hline
\end{tabular}

* Densidade básica determinada nas amostras de pisos conforme ABNT 11941 (Associação Brasileira de Normas Técnicas 2003), após a determinação dos parâmetros colorimétricos. ${ }^{1}$ Fonte: Costa et al. (2021)

Para cada espécie, foram selecionados vinte corpos de prova com as dimensões $12,7 \times 27,94 \times 2$ $\mathrm{cm}$, obtidos de pisos de madeira maciça da madeira de cerne, tipo assoalho, retirada na própria linha de produção, cujo desdobro foi do tipo tangencial. Posteriormente, os corpos de prova foram acondicionados em sala climatizada, com temperatura de $20 \pm 2{ }^{\circ} \mathrm{C}$ e umidade relativa do ar de $65 \pm 5 \%$, para posterior avaliação.

Para a caracterização colorimétrica, os dados foram obtidos com auxílio do colorímetro CR-410 Chroma Meter. Antes das análises, realizou-se a calibração do colorímetro, com o auxílio de duas referências fornecidas pelo equipamento, uma com $\mathrm{L}^{*}=100$ (branco total) e outra com $\mathrm{L}^{*}=0$ (preto total). Posteriormente, as medidas foram realizadas por contato direto da superfície da amostra com o aparelho, em temperatura ambiente. A abertura do sensor era de $5 \mathrm{~mm}$, a fonte de luz (iluminante) C era composta por uma lâmpada de xenônio, observador padrão suplementar de $10^{\circ}$ e área de iluminação igual a $53 \mathrm{~mm}$ de diâmetro.

Foram realizadas cinco leituras por corpo de prova, na face tangencial, obtendo-se os parâmetros colorimétricos L* (luminosidade), a* (coordenada cromática verde-vermelho) e $b^{*}$ (coordenada cromática azul-amarelo), baseado no sistema CIEL*a*b* (Comission International de L'Eclairage). Para o cálculo dos parâmetros C (saturação) e h* (ângulo de tinta), foram utilizadas as Equações 1 e 2.

$\mathrm{C}=\sqrt{(}\left(a *^{2}+\mathrm{b} *^{2}\right)$

$$
h *=\tan ^{-1}\left(\frac{b *}{a *}\right)
$$

Para a definição do padrão de cor dos pisos de madeira, foi utilizada uma tabela de cores com variações numéricas dos parâmetros analisados ( $\mathrm{L}^{*}$, $a^{*}, b^{*}, C$ e h*), proposta por Camargos e Gonçalez (2001). Posteriormente, os valores dos parâmetros $L^{*}, a^{*}$ e $b^{*}$ foram transformados em RGB (abreviatura do sistema de cores aditivas formado por Vermelho (Red), Verde (Green) e Azul (Blue)), para a construção de uma figura representativa da caracterização colorimétrica dos pisos.

Os dados foram submetidos aos testes de Shapiro-Wilk, para testar a normalidade dos erros, e de Bartlett, para testar a homogeneidade das variâncias. Em seguida, os resultados foram submetidos à análise de variância (ANOVA), para verificação das diferenças entre os tratamentos avaliados. Quando estabelecidas diferenças significativas entre eles, aplicou-se o teste de Tukey $(p \leq 0,05)$. Os parâmetros colorimétricos dos pisos foram correlacionados com a densidade básica da madeira, por meio da correlação de Pearson. As análises estatísticas foram realizadas no software livre R (R Core Team 2018).

\section{Resultados e discussão}

Os resultados obtidos para os parâmetros colorimétricos dos pisos maciços de madeiras das cinco espécies avaliadas estão apresentados na Tabela 2 e na Figura 1.

Tabela 2. Valores médios e coeficiente de variação (\%) dos parâmetros colorimétricos dos pisos das madeiras tropicais amazônicas avaliadas

\begin{tabular}{cccccc}
\hline \multirow{2}{*}{ Espécie } & \multicolumn{5}{c}{ Parâmetros Colorimétricos } \\
\cline { 2 - 5 } & $\mathrm{L}^{*}$ & $\mathrm{a}^{*}$ & $\mathrm{~b}^{*}$ & $\mathrm{C}$ & $\mathrm{h}^{*}$ \\
\hline Cumaru & $54,43 \mathrm{c}^{(6,97)}$ & $11,79 \mathrm{~b}^{(13,36)}$ & $22,97 \mathrm{c}^{(8,75)}$ & $25,81 \mathrm{~b}^{(7,30)}$ & $62,82 \mathrm{bc}^{(2,95)}$ \\
\hline Ipê & $47,43 \mathrm{~d}^{(7,34)}$ & $6,50 \mathrm{~d}^{(35,16)}$ & $17,74 \mathrm{e}^{(12,41)}$ & $18,89 \mathrm{~d}^{(11,89)}$ & $69,86 \mathrm{a}^{(8,24)}$ \\
\hline Jatobá & $59,16 \mathrm{~b}^{(5,83)}$ & $13,52 \mathrm{a}^{(9,40)}$ & $24,96 \mathrm{~b}^{(6,70)}$ & $28,38 \mathrm{a}^{(4,97)}$ & $61,56 \mathrm{c}^{(2,82)}$ \\
\hline Muiracatiara & $63,08 \mathrm{a}^{(7,54)}$ & $13,22 \mathrm{a}^{(10,96)}$ & $26,16 \mathrm{a}^{(6,30)}$ & $29,31 \mathrm{a}^{(6,15)}$ & $63,18 \mathrm{bc}^{(3,00)}$ \\
\hline Sucupira-preta & $53,97 \mathrm{c}^{(4,31)}$ & $10,33 \mathrm{c}^{(10,39)}$ & $21,07 \mathrm{~d}^{(9,67)}$ & $23,47 \mathrm{c}^{(6,93)}$ & $63,88 \mathrm{~b}^{(2,14)}$
\end{tabular}

Médias seguidas pela mesma letra, dentro da mesma coluna, não diferem estatisticamente (p $\leq 0,05)$, pelo teste de Tukey; e (..) coeficiente de variação (\%). 
Para Martins et al. (2015), quando há homogeneidade da cor em um lote de madeira beneficiada, maior é sua aceitação seja para o vendedor, seja para o consumidor. No entanto, a variação de cor também pode ser um diferencial, dependendo das preferências do consumidor.

No caso específico de pisos de madeira, a amplitude do ambiente será afetada: um piso com grande variação de cor deixa o ambiente com aspecto reduzido; já a uniformidade cria a sensação de maior amplitude do espaço e oferece maior neutralidade, já que o piso ganha menor destaque.

Para o parâmetro L* (Tabela 2 e Figura 1A), foram constatadas diferenças significativas entre as espécies $(p \leq 0,05)$, exceto para cumaru e sucupirapreta que foram estatisticamente iguais. Os valores encontrados para este parâmetro estão de acordo com os observados por Melo et al. (2019) ao realizarem a caracterização colorimétrica de 18 espécies tropicais amazônicas.
Conforme a classificação proposta por Camargos e Gonçalez (2001), os pisos de cumaru, ipê e sucupira-preta, por apresentarem valores médios para $\mathrm{L}^{*}$ inferiores a 56 , enquadraram-se como pisos escuros. Já os pisos de muiracatiara e jatobá pelo fato dos valores médios serem superiores a 56, seriam claros por essa classificação, no entanto, pelos valores próximos ao limite estabelecido, enquadram-se como menos escuros.

Em relação à luminosidade, pisos escuros têm o efeito da redução do ambiente em que estão instalados e trazem a sensação de maior conforto, sendo indicados para dormitórios e salas de estar, escritórios ou ambientes com poucos móveis. Já os pisos claros contribuem para o aspecto de um ambiente mais amplo, além de aproveitarem melhor a luz natural, sendo recomendado para ambientes como salas de jantar, salas de espera ou lojas comerciais.

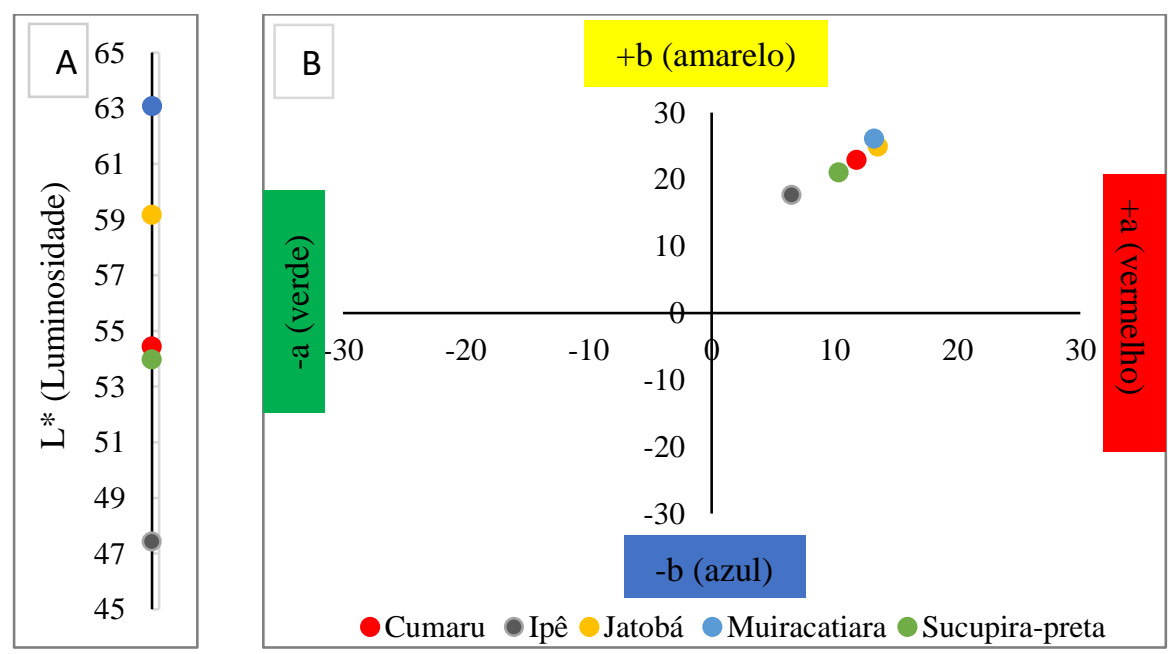

Figura 1. (A) Luminosidade e (B) parâmetros a* (eixo azul-amarelo) e b* (eixo verde-vermelho) dos pisos madeiras tropicais amazônicas avaliadas.

Para o parâmetro a* (Tabela 2 e Figura 1B), verificou-se que os valores médios para a madeira dos pisos de todas as espécies, situaram-se no lado vermelho do eixo verde-vermelho, assim como verificado por Melo et al. (2019) em estudo com 18 espécies tropicais e por Zaque et al. (2019) ao avaliarem a madeira das principais espécies comercializadas pelo estado de Mato Grosso. As espécies muiracatiara e jatobá apresentaram os maiores valores para este parâmetro, e não diferiram estatisticamente entre si, enquanto para o ipê foi observado o menor valor médio (Tabela 2 ).

As espécies de cumaru e sucupira-preta apresentaram valores intermediários e foram estatisticamente diferentes entre si e das demais espécies $(\mathrm{p} \leq 0,05)$.

Para o parâmetro b* (Tabela 2 e Figura 1B), constatou-se que os valores médios para a madeira dos pisos de todas as espécies situaram-se no lado amarelo do eixo azul-amarelo. Todas as espécies avaliadas diferiram estatisticamente entre si $(\mathrm{p} \leq 0,05)$, em relação a esse parâmetro, com menor valor médio para o ipê e o maior para a espécie muiracatiara. Zaque et al. (2019) ao realizarem a caracterização colorimétrica das trinta principais espécies de madeira comercializadas no Mato Grosso encontraram valores positivos, com variação de 14,78 a 30,63 , demonstrando o padrão das madeiras amazônicas em serem mais "avermelhadas", assim como verificado para as madeiras avaliadas.

Os parâmetros $a^{*}$ e $b^{*}$ que representam a tonalidade, exercem influência marcante na caracterização de cor das espécies. Para todas as madeiras avaliadas, a influência do parâmetro $b^{*}$ foi maior, o que evidencia que a cor da madeira dessas 


\section{Costa et al.}

espécies situa-se mais próximo do eixo amarelo (Figura 1B). A espécie ipê apresentou o maior valor para o ângulo de tinta $\left(\mathrm{h}^{*}\right)$, seguido das demais espécies que foram estatisticamente iguais $(\mathrm{p} \leq 0,05)$ (Tabela 2).

O ângulo de tinta $\left(\mathrm{h}^{*}\right)$ maior que $60^{\circ}$ (Tabela 2) confirma a proximidade das cores das espécies com esse eixo, o que indica maior tendência das madeiras avaliadas serem amareladas. Melo et al. (2019) confirmaram essa tendência para as espécies tropicais ao verificarem que $83 \%$ das espécies avaliadas em seu trabalho apresentaram ângulo de tinta $\left(h^{*}\right)$ maior que $60^{\circ}$.

Todas as espécies avaliadas podem ser consideradas madeiras de cor com baixa saturação (C), por apresentarem valores inferiores a 30 (Tabela 2), ou seja, possuem maior concentração de cinza na composição da cor (Camargos e Gonçalez 2001) Os maiores valores médios de saturação foram observados para as espécies muiracatiara e jatobá, que não diferiram estatisticamente entre si $(\mathrm{p} \leq 0,05)$, e o menor para o ipê. Como o parâmetro $\mathrm{C}$ é influenciado pelos parâmetros $\mathrm{a}^{*} \mathrm{e} \mathrm{b}^{*}$, à medida que os valores dessas duas variáveis aumentam, há o acréscimo nos valores da saturação, conforme verificado na Figura 1B.

Os valores médios dos parâmetros colorimétricos $\mathrm{a}^{*}, \mathrm{~b}^{*}$ e C (Tabela 2) dos pisos de madeira maciça indicam uma preferência do mercado consumidor por madeiras mais escuras, amareladas e com baixa saturação, uma vez que os pisos dessas espécies são os mais comercializados pela indústria madeireira deste estudo.

Na Figura 2 estão apresentadas as cores dos pisos das madeiras das espécies avaliadas e a representação em RGB, na qual foi possível associar a classificação colorimétrica para madeiras tropicais com as cores obtidas em RGB.

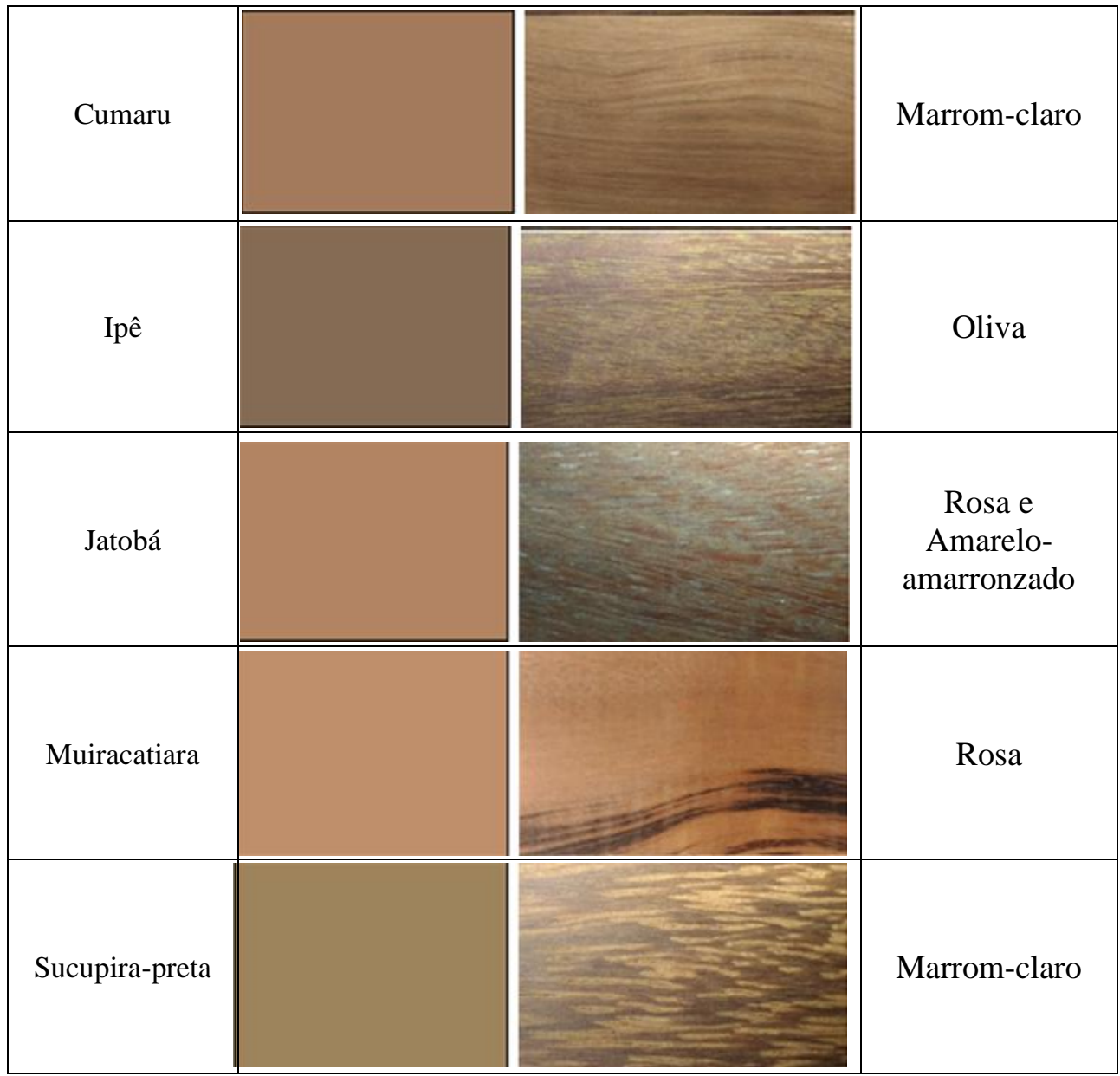

Figura 2. Representação das cores dos pisos das madeiras tropicais amazônicas em RGB; e suas respectivas imagens e classes de cor atribuídas.

Os pisos das madeiras de cumaru, ipê e sucupira-preta apresentaram-se mais escuros, conforme valores de $\mathrm{L}^{*} \mathrm{e} \mathrm{h} \mathrm{h}^{*}$ (Tabela 2). Já os de jatobá e de muiracatiara são menos escuros, devendo ser ressaltado que o piso de muiracatiara apresentou o maior valor do parâmetro $\mathrm{L}^{*}$, sendo o mais claro entre as cinco espécies avaliadas (Tabela 2).

Os pisos de madeira das espécies cumaru e sucupira-preta foram caracterizados como marromclaros, de acordo com a classificação de Camargos 


\section{Costa et al.}

et al. (2001) e assemelham-se visualmente (Figura 2). A classificação da madeira de cumaru se assemelham ao reportado por Zaque et al. (2019) que classificaram a madeira dessa espécie como marromclaro; enquanto que Melo et al. (2019) e Sousa et al. (2019) classificaram como oliva-amarelada. Para a madeira de Bowdichia nítida, popularmente classificada como sucupira, Sousa et al. (2019) classificaram-na como marrom avermelhado. Classificações próximas, evidenciando um padrão para essas espécies.

A cor dos pisos da madeira de muiracatiara foi caracterizada como rosa, diferindo da coloração observada por Silva et al. (2017) e Zaque et al. (2019), que classificaram a madeira dessa espécie como amarelo-amarronzado e de Sousa et al. (2019), que classificaram como marrom-oliva. Essa diversidade por ser atribuída as listras típicas dessa espécie (Figura 2), que podem influenciar nos parâmetros colorimétricos

A cor dos pisos da madeira de jatobá enquadrou-se em duas classes de cores, rosa e amarelo-amarronzado, distinto do observado por Melo et al. (2019) que enquadraram também em duas classes: amarelo claro e oliva-amarronzado; e Silva et al. (2017), que classificaram a madeira como marrom-avermelhada e Sousa et al. (2019) que classificaram como oliva-amarelada. Já Zaque et al.
(2019) classificaram a madeira de jatobá como amarelo-amarronzado, assim como o presente trabalho.

Já o piso de ipê foi caracterizado como oliva, corroborando o observado por Silva et al. (2017) e Zaque et al. (2019). Destaca-se que a madeira de ipê foi a que mais se distinguiu do padrão de cores entre as espécies avaliadas (Figura 2).

As diferenças entre os valores verificados neste estudo e os descritos na literatura podem estar relacionadas a variações genéticas e ambientais, ou de processamento, como o tipo de desdobro (radial ou tangencial), que alteram as características organolépticas e químicas da madeira. A cor da madeira pode ser alterada pela variação no teor de umidade e temperatura, deterioração por organismos xilófagos ou por reações fotoquímicas (Camargos e Gonçalez 2001) Essas variações ressaltam a importância da avaliação da cor como um critério auxiliar de classificação de qualidade dos pisos de madeira, além de sua determinação visando a formação de grupos de madeira em relação a sua classificação de cor.

Na Figura 3 é apresentada a correlação entre os parâmetros colorimétricos dos pisos $\left(\mathrm{L}^{*}, \mathrm{a}^{*}, \mathrm{~b}^{*}\right.$, $\mathrm{C}$ e $\left.\mathrm{h}^{*}\right)$ e a densidade básica da madeira das espécies avaliadas.

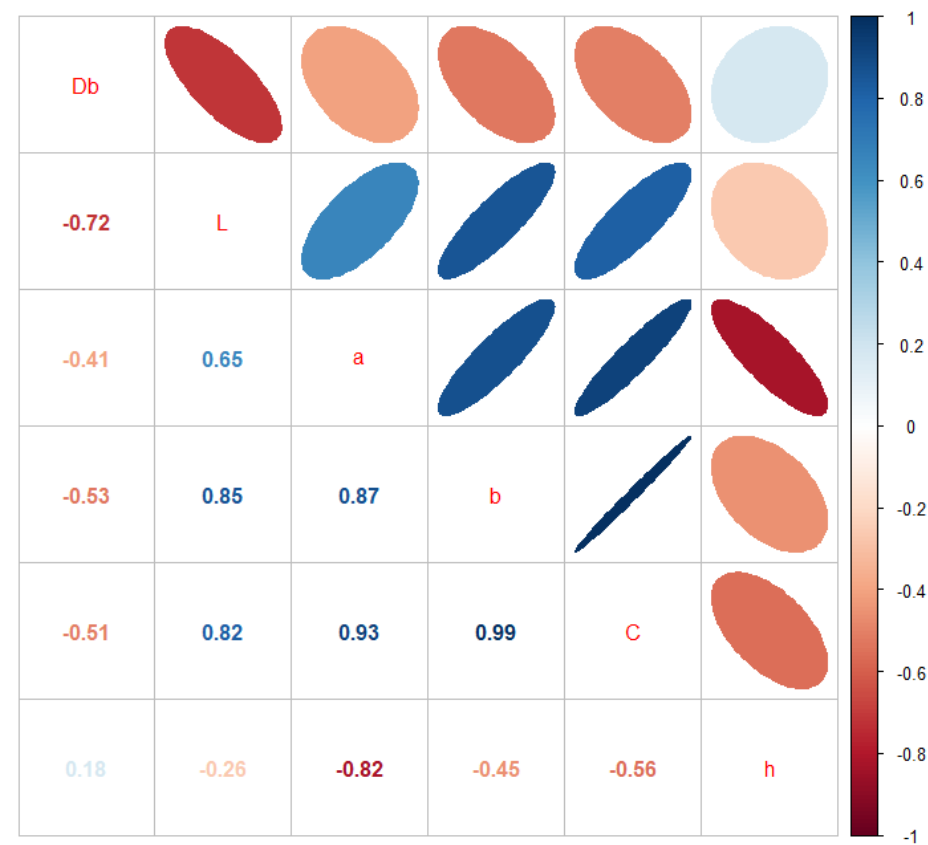

Figura 3. Matriz de Correlação de Pearson entre os parâmetros colorimétricos (L*, a*, b*, C e h*) e a densidade básica $(\mathrm{Db})$ dos pisos de madeira das espécies avaliadas $(\mathrm{p} \leq 0,05)$.

A densidade básica da madeira teve uma forte correlação negativa $(-0,72)$ com o parâmetro $L^{*}$ (Figura 3). Assim, pode-se afirmar que madeiras mais claras são mais leves e as mais escuras são mais pesadas. Essa relação inversa de densidade e luminosidade também foi observada por Sousa et al.
(2019) e Zaque et al. (2019) avaliando madeiras tropicais, Amorim et al. (2013) ao avaliarem madeiras de Pinus e Eucalyptus e Garcia et al. (2016) ao avaliarem madeira de Tectona grandis.

Neste estudo, essa relação pode ser verificada para os pisos das madeiras de cumaru e ipê, que 
apresentaram menores valores médios do parâmetro L (Tabela 2) e maiores densidades básicas (Tabela 1), sendo essas madeiras classificadas como escuras e pesadas. Enquanto que o piso da madeira de muiracatiara, que apresentou o menor valor médio de densidade (Tabela 1) e foi classificado como o mais claro (Tabela 2).

Pisos confeccionados com madeira de maior densidade apresentam menor deterioração quando submetidas ao uso, como riscos, danos por quedas de objetos, desgaste por elementos abrasivos, tráfego de pessoas ou arraste de objetos (Blanco et al. 2015, Oliveira et al. 2019). Assim, o parâmetro luminosidade $\left(\mathrm{L}^{*}\right)$ pode ser utilizado para uma seleção ou classificação prévia das madeiras em relação a densidade básica, permitindo a comparação entre madeiras com valores distintos de densidade, pois os parâmetros colorimétricos da madeira podem ser obtidos de forma rápida, por meio de aparelhos portáteis. No entanto, em razão dos diversos fatores que afetam a densidade, essa deve ser determinada, assim como a realização de ensaios de simulação de uso para pisos para melhor definição do ambiente de utilização desses pisos.

A densidade básica apresentou correlação moderada negativa com os parâmetros $a^{*}$ e b* (Figura 3), ou seja, quanto maior a densidade, menor os valores para estes parâmetros. Zaque et al. (2019) ao avaliarem 30 madeiras tropicais verificaram para a densidade uma correlação positiva fraca $(+0,39)$ com o parâmetro a* (eixo verde-vermelho) e negativa fraca $(-0,22)$ para o parâmetro $b^{*}$ (eixo azul-amarelo), enquanto que (Sousa et al. 2019) ao avaliarem 10 madeiras tropicais encontraram uma forte correlação positiva $(+0,80)$ para o parâmetro $a^{*}$ e uma correlação bem fraca positiva $(0,11)$ para o parâmetro $b^{*}$ em relação a densidade.

Portanto, verifica-se que não há um padrão de correlação da densidade com os parâmetros $a^{*} \mathrm{e} \mathrm{b*}$, diferentemente do que ocorre com o parâmetro L* (luminosidade). Isso acontece porque a pigmentação pode ser influenciada pela composição química da madeira, principalmente pelos extrativos, que são componentes que aparecem em pequenas quantidades e tem estrutura química de baixo peso molecular, mas de grande importância na determinação da cor da madeira (Bonfatti Júnior et al. 2018). De acordo com Barreto et al. (2009), os extrativos de espécies arbóreas tropicais brasileiras são muito diversos, tanto em quantidade quanto em natureza química.

A luminosidade (L*) apresentou correlação positiva com os parâmetros $\mathrm{a}^{*}, \mathrm{~b}^{*}$ e $\mathrm{C}$ e negativa com o parâmetro h* (Figura 3). Zaque et al. (2019) encontraram correlação positiva do parâmetro $\mathrm{L}^{*}$ com b*, C e h* e negativa com o parâmetro a*, enquanto que Sousa et al. (2019) observaram correlação positiva de $\mathrm{L}^{*}$ com b* e C, e negativa com $a^{*} \mathrm{e} h *$. Esses resultados indicam que madeiras que apresentem maior valor de $b^{*}$ (mais amareladas), tendem a apresentar maior luminosidade (mais claras), no entanto para o parâmetro a* não há um padrão associado à luminosidade ( $\left.\mathrm{L}^{*}\right)$.

Conforme resultados encontrados, para a adoção dos parâmetros colorimétricos como ferramenta para determinação da qualidade de pisos maciços de madeira, é necessária a realização de mais estudos, contemplando maior número de amostras (pisos) e espécies, além de relacionar os parâmetros colorimétricos com resultados dos ensaios de simulação de uso de pisos.

\section{Conclusões}

Os pisos de madeira das espécies cumaru, ipê e sucupira-preta são escuros e os de jatobá e muiracatiara, menos escuros e em geral, os pisos de madeira têm tendência de serem mais amarelos que vermelhos.

Há uma correlação inversa entre a densidade básica e a luminosidade ( $\mathrm{L}^{*}$ ) das madeiras avaliadas. Portanto, esse parâmetro colorimétrico pode ser utilizado pelas indústrias como critério auxiliar na seleção de madeiras destinadas à produção de pisos, considerando a relação com a densidade básica da madeira e sua influência na qualidade dos pisos.

\section{Agradecimentos}

Os autores agradecem o apoio da Coordenação de Aperfeiçoamento de Pessoal de Nível Superior (CAPES); do Conselho Nacional de Pesquisa e Desenvolvimento (CNPq); da Fundação de Amparo à Pesquisa do Estado de Mato Grosso (FAPEMAT); da empresa Brasil Tropical Pisos; do Programa de Pós-Graduação em Ciências Florestais e Ambientais; do Laboratório de Ciência e Tecnologia de Alimentos da Universidade Federal de Mato Grosso.

\section{Referências}

Amorim PGR, Gonçalez JC ,Camargos JAA (2013). Propriedades da madeira de Pinus caribaea e Eucalyptus grandis estimadas por colorimetria. Cerne 19(3): 461-466

Associação Brasileira da Indústria de Madeira Processada Mecanicamente ABIMCI (2019). Estudo setorial 2019: ano base 2018. 161

Associação Brasileira de Normas Técnicas ABNT (2003). NBR 11941: madeira: determinação da densidade básica. Rio de Janeiro, ABNT: 6 .

Barreto CCK, Pastore TCM (2009). Resistência ao intemperismo artificial de quatro madeiras tropicais: o efeito dos extrativos. Ciência Florestal 19(1): 2330 
Barros SVdS, Muniz GIBd, Matos JLMd (2014). Caracterização colorimétrica das madeiras de três espécies florestais da Amazônia. Cerne 20(3): $337-$ 342.doi: 10.1590/01047760201420031421.

Blanco FJ, Silva JRM, Braga PP, Lima JT, Trugilho PF (2015). Simulação em serviço de pisos de madeira jovem de Tectona grandis. Revista Matéria 20(4): $\quad$ 1048-1060.doi: $\quad$ 10.1590/S1517707620150004.0107.

Bonfatti Júnior EA, Lengowski EC (2018). Colorimetria aplicada à ciência e tecnologia da madeira. Pesquisa Florestal Brasileira 38: 1-13.doi: 10.4336/2018.pfb.38e201601394.

Camargos JA, Gonçalez JC (2001). A colorimetria aplicada como instrumento na elaboração de uma tabela de cores de madeira. Brasil Florestal 71: 1-12

Costa ACS, Oliveira AC, Pereira BLC, Silva JRMd, Oliveira MBd, Xavier CN (2021). Quality of Tropical Hardwood Floors. Revista Árvore 45.doi: 10.1590/1806-908820210000003.

Garcia RA, Marinonio GB (2016). Variação da cor da madeira de Teca em função da densidade e do teor de extrativos. Floresta e Ambiente 23(1): 124134.doi: 10.1590/2179-8087.035313.

Gierlinger N, Jacques D, Grabner M, Wimmer R, Schwanninger M, Rozenberg P, Pâques L (2004). Colour of larch heartwood and relationships to extractives and brown-rot decay resistance. Trees Structure and Function 18(1): 102-108.doi: 10.1007/s00468-003-0290-y.

Martins MDF, Beltrame R, Delucis RDA, Gatto DA, De Cademartori PHG, Dos Santos GA (2015). Colorimetria como ferramenta de agrupamento de madeira de clones de eucalipto. Pesquisa Florestal Brasileira 35(84): $\quad$ 443-449.doi: 10.4336/2015.pfb.35.84.929.

Melo RRd, Stangerlin DM, Del Menezzi CHS, Castro VGd, Souza MJCd, Medeiros DTd (2019). Grouping of commercial tropical timber using wood color. Revista de Ciências Agrárias 62: 1-6.doi: 10.22491/rca.2019.3108.

Moya R, Calvo-Alvarado J (2012). Variation of wood color parameters of Tectona grandis and its relationship with physical environmental factors. Annals of Forest Science 69(8): 947-959.doi: 10.1007/s13595-012-0217-0.

Oliveira MBd, Silva JRMd, Hein PRG, Lima JT (2019). Establishment of quality classes for hardwood floorings by simulated use. Cerne 25(1): 105-109.doi: 10.1590/01047760201925012618.

R Core Team R (2018). R: A language and environment for statistical computing. Vienna, Austria, R Foundation for Statistical Computing.

Silva RAFd, Setter C, Mazette SS, Melo RRd, Stangerlin DM (2017). Colorimetria da madeira de trinta espécies tropicais. Revista Ciência da Madeira 8(1): 36-41.doi: 10.12953/2177-6830/rcm.v8n1p3641.

Silva SB, Andrade JKB, Arantes MDC, Silva JGM, Brocco VF, Paes JB (2019). Color variability and relationship with basic density and extractive content of Calycophyllum spruceanum wood. Floresta 49(4): 803-812.doi: 10.5380/rf.v49 i4.59374.

Sousa WCSe, Barbosa LdJ, Soares AAV, Goulart SL, Protásio TdP (2019). Wood colorimetry for the characterization of Amazonian tree species: a subsidy for a more efficient classification. Cerne 25(4): 451-462.doi: 10.1590/01047760201925042650.

Zaque LAM, Melo RRd, Souza ECd, Serenine Junior L (2019). Caracterização colorimétrica e da massa específica das madeiras comercializadas no Mato Grosso. Revista Ciência da Madeira 10(3): 236-246.doi: 10.12953/2177-6830/rcm.v10n3p236246. 